\title{
Erratum to: A phylogenetic study of subtribe Otachyriinae (Poaceae, Panicoideae, Paspaleae)
}

\author{
J. M. Acosta $\cdot$ M. A. Scataglini $\cdot$ R. Reinheimer • \\ F. O. Zuloaga
}

Published online: 26 June 2014

(c) Springer-Verlag Wien 2014

\section{Erratum to: Plant Syst Evol \\ DOI 10.1007/s00606-014-1034-8}

In the original publication of the article, GenBank accession numbers for 21 new sequences obtained for this work, were not listed in Appendix 1. With this erratum, the authors have provided taxa, voucher specimens from which DNA was extracted for sequencing, and its corresponding GenBank accession numbers:

Anthaenantia rufa (Elliott) Schult., KJ719510

Henderson 96820

Anthaenantia texana Kral, Kral $93287 \quad$ KJ719511

Anthaenantia villosa (Michx.) P. Beauv., Kral KJ719512 93319

Hymenachne amplexicaulis (Rudge) Nees, KJ719513

Morrone 5190

Hymenachne pseudointerrupta Müll. Hal., KJ719514

Beusehom 4230

Otachyrium aquaticum Send. \& Soderstr., KJ719515

Mori 12348

Otachyrium grandiflorum Send. \& Soderstr., KJ719516

Fonseca \& Alvarenga 2206

Otachyrium piligerum Send. \& Soderstr., KJ719517

Rodriguez 157 $\underline{\text { Otachyrium pterygodium }}$ (Trin.) Pilg., $\quad$ KJ719518 Andersson 8880

Otachyrium seminudum Hack. ex Send. \& KJ719519 Soderstr., Walter 904

Otachyrium succisum (Swallen) Send. \& KJ719520

Soderstr., Jansen-Jacobs 2723

Panicum auritum J. Presl ex Nees, Olsen 144 KJ719521

Panicum harleyi D. Salariato, Morrone \& KJ719522

Zuloaga, Harley 5480

Panicum hemitomon Schult., Jones 23315 KJ719523

Panicum leptachne Döll, Longhi-Wagner KJ719524 9511

Panicum polygonatum Schrad., Morrone KJ719525 4269

$\underline{\text { Panicum stagnatile }}$ Hitchc. \& Chase, Harmel KJ719526 8404

Panicum stevensianum Hitchc. \& Chase, KJ719527

Ekman 1088

Steinchisma cuprea (Hitchc. \& Chase) W.V. KJ719528 Br., Beetle 7708

Steinchisma exiguiflora (Griseb.) W.V. KJ719529 Br., Zuloaga 9580

Steinchisma stenophylla (Hack.) Zuloaga \& KJ719530 Morrone, Veredao da Silva 3459

The online version of the original article can be found under doi:10.1007/s00606-014-1034-8.

J. M. Acosta $(\bowtie) \cdot$ M. A. Scataglini · R. Reinheimer .

F. O. Zuloaga

Instituto de Botánica Darwinion, Labardén 200,

Casilla de Correo 22, B1642HYD San Isidro, Buenos Aires,

Argentina

e-mail: jacosta@darwin.edu.ar 